\title{
Article \\ Clinical and Instrumental TMJ Evaluation in Children and Adolescents with Juvenile Idiopathic Arthritis: A Case-Control Study
}

\author{
Michele D'Attilio ${ }^{1, *}\left(\mathbb{D}\right.$, Beatrice Di Carlo ${ }^{1}\left(\mathbb{D}\right.$, Francesco Caroccia ${ }^{1,2} @$, Francesco Moscagiuri ${ }^{1}$, \\ Debora Mariarita d'Angelo ${ }^{3}$, Francesco Chiarelli ${ }^{3}$, Felice Festa ${ }^{1}$ and Luciana Breda ${ }^{3}$ \\ 1 Department of Innovative Technologies in Medicine \& Dentistry, University of Chieti-Pescara, \\ 66100 Chieti, Italy; bdc.dicarlo@gmail.com (B.D.C.); fcaroccia20@gmail.com (F.C.); \\ francesco.moscagiuri@unich.it (F.M.); felice.festa@unich.it (F.F.) \\ 2 Department of Surgery and Translational Medicine, University of Florence, 50127 Firenze, Italy \\ 3 Paediatric Rheumatology Unit, Department of Paediatrics, University of Chieti-Pescara, 66100 Chieti, Italy; \\ deboramrdangelo@gmail.com (D.M.d.); chiarelli@unich.it (F.C.); luciana.bredach@gmail.com (L.B.) \\ * Correspondence: michele.dattilio@unich.it; Tel.: +39-335-7489425
}

Citation: D'Attilio, M.; Di Carlo, B.; Caroccia, F.; Moscagiuri, F.; d'Angelo, D.M.; Chiarelli, F.; Festa, F.; Breda, L. Clinical and Instrumental TMJ Evaluation in Children and Adolescents with Juvenile Idiopathic Arthritis: A Case-Control Study. Appl. Sci. 2021, 11, 5380. https:// doi.org/10.3390/app11125380

Academic Editors:

Alessandra Lucchese, Dario Bertossi and Riccardo Nocini

Received: 10 May 2021

Accepted: 8 June 2021

Published: 10 June 2021

Publisher's Note: MDPI stays neutral with regard to jurisdictional claims in published maps and institutional affiliations.

Copyright: (c) 2021 by the authors. Licensee MDPI, Basel, Switzerland. This article is an open access article distributed under the terms and conditions of the Creative Commons Attribution (CC BY) license (https:/ / creativecommons.org/licenses/by/ $4.0 /)$.
Abstract: To investigate temporomandibular joint (TMJ) involvement signs such as muscle pain, the ratio of masseter and temporal muscle activity, mouth opening width and jaw deviation during mouth opening in children and adolescents with juvenile idiopathic arthritis (JIA), a group of 32 subjects with JIA and a control group of 32 healthy subjects were evaluated. Data were collected clinically by muscle palpation (masseters, anterior temporalis and sternocleidomastoids) and instrumental analysis (electromyography and kinesiography). Higher pain was registered in the masseter and sternocleidomastoid muscles on both sides and in the right anterior temporalis in the JIA group compared to the control group $(p<0.05)$. Electromyography showed no statistically significant difference in the frequency of the pathological ratio of masseter and temporal muscle activity $(\mathrm{MM} / \mathrm{TA}<1)$ both in the JIA group and in the control group. Kinesiography showed a statistically significant difference in mouth opening width and jaw deviation during mouth opening between the groups $(p<0.05)$ : JIA subjects showed lower mouth opening values and wider deviation on mouth opening; 29 out of 32 JIA subjects showed jaw deviation towards the right side. JIA affects the TMJ, causing myalgia in the head and neck muscles, a reduction in mouth opening width and an increase in jaw deviation during mouth opening.

Keywords: juvenile idiopathic arthritis; temporomandibular joint; electromyography; kinesiography

\section{Introduction}

Juvenile idiopathic arthritis (JIA) is a broad term used to describe a heterogeneous group of diseases characterized by arthritis of unknown origin beginning before the 16th birthday and persisting for at least 6 weeks [1]. JIA is the most common chronic rheumatic disease in childhood. The incidence and prevalence have ranges between 1.6 and 23 new cases per 100,000 children and 3.8 and 400 cases per 100,000 children, respectively [2]. JIA is characterized by persistent inflammation of at least one of the several joints in the human body $[3,4]$. Despite the fact that recent studies suggested the influence of both genetic and environmental factors, thus defining a heterogeneous illness, the main cause of JIA is still poorly understood [5,6]. A main factor in the development and regulation of the immune system, the human leukocyte antigen (HLA) seems to be primary involved [7], resulting in chronic inflammation development [8].

The JIA classification proposed by the International League of Associations for Rheumatology (ILAR) in 1995 [9] and then revised in 2001 [10] is based on clinical and laboratory parameters evaluated in the first 6 months of disease. The ILAR classification consists of seven different categories: systemic arthritis, oligoarthritis (persistent or extended), 
polyarthritis rheumatoid factor (RF)-positive, polyarthritis RF-negative, enthesitis-related arthritis (ERA), psoriatic arthritis (PsA) and undifferentiated arthritis, the last category including those patients who do not fit any criteria or fit more than one. These criteria, however, are currently under revision with a new ongoing study by the Paediatric Rheumatology International Trials Organisation (PRINTO) [11].

The clinical manifestation of JIA is unpredictable: in some cases, the disease course is mild, like a self-limiting disease; other patients could suffer symptomatic joint erosion with an unremitting pattern [2]. Clinical and laboratory records are used to help diagnose and classify the disease, but magnetic resonance imaging (MRI) is the gold standard technique for the study of JIA [4,12]. Musculoskeletal ultrasound and magnetic resonance imaging resulted in being more sensitive than clinical examination and radiography to detect joint involvement [12].

JIA has a close relationship with the stomatognathic system. High levels of caries and gingival inflammation have been found in adolescents affected by JIA due to increased systemic inflammatory indexes, sugar-containing medications and reduced jaw movements and motor skills [13]. Moreover, about 55\% of JIA patients show signs and symptoms of temporomandibular joint (TMJ) involvement [14].

TMJ arthritis can affect dentofacial growth and mandibular function [15-17]. Kjellberg in 1995 [16] reported that the dentofacial morphology in JIA children is characterized by a smaller, more retrognathic and steeper inclined mandible compared to that of healthy children with ideal occlusion. The author [16] concluded that dentofacial growth, chewing movement restriction, decreased bite force and low mouth opening capacity are frequent consequences of condylar lesions. As reported in the literature, about $26 \%$ of patients with JIA show symptoms related to the masticatory system and mostly pain during mandibular function and stiffness at mouth opening [18]. Restricted maximal voluntary mouth opening is, above all, the most frequent clinical sign [14,18]. Pain during jaw excursion and restricted mouth opening have been proposed as predictor factors for TMJ involvement; however, the sensitivity of these clinical signs resulted in being too low [14].

Several studies have investigated TMJ involvement in JIA patients with instrumental equipment such as stereophotogrammetrically with 3D photographs [19], power Doppler ultrasound (US) [20], 3D surface scans [21], CBCT [22] and orthodontic orthopantomograms [23]. Other studies defined clinical protocols for orofacial examination in JIA children $[15,24]$. Recent systematic reviews concluded that there are current low levels of evidence, and that the heterogeneity of the studies does not allow identifying clinical findings that could accurately predict TMJ arthritis $[25,26]$.

The objective of the current study is to test clinical and instrumental methods as reliable, non-invasive functional prognostic indicators of possible TMJ involvement in patients with JIA. The outcomes investigated are the following: muscle pain, the ratio of masseter and temporal muscle activity, mouth opening width and jaw deviation during mouth opening. The methods tested are as follows: (a) palpation of head and neck muscles, (b) electromyography (EMG) and (c) kinesiography. The null hypothesis to be tested is that there are no significant differences observed in pain during muscle palpation, in mouth opening width and deviation on mouth opening during kinesiography and in the frequency of the pathologic ratio during electromyography between case and control groups.

\section{Materials and Methods}

\subsection{Subjects}

The study was reported using the STROBE statement for observational studies [27].

Originally, the test group included 80 patients recruited from March 2019 until November 2019 in the Department of Paediatrics of the University of Chieti, Italy. The authors confirmed the criteria of the Helsinki Declaration and the ICH Guideline for Good Clinical Practice. Subjects' parents gave informed consent at the beginning of the study before confirming that they were fit for this study. Subjects could withdraw from this work for any reason and without prejudice at any time. The protocol was reviewed by the Local 
Ethical Committee of the University of Chieti (protocol no: MGB_AIG). After applying the inclusion/exclusion criteria described in Table 1, only 32 subjects (26 female and 6 male, average age 13 years \pm 2 ) were finally recruited for the study.

Table 1. Inclusion/exclusion criteria.

\begin{tabular}{cc}
\hline Inclusion Criteria & Exclusion Criteria \\
\hline $\begin{array}{c}\text { Patients with juvenile idiopathic arthritis } \\
\text { diagnosed at least six months before by the } \\
\text { Paediatric Department of the SS. Annunziata } \\
\text { Hospital in Chieti, Italy }\end{array}$ & $\begin{array}{l}\text { Incomplete medical history } \\
\text { Age between } 5 \text { and 20 years old } \\
\text { No contemporary or previous } \\
\text { orthodontic treatment }\end{array}$ \\
$\begin{array}{c}\text { Compliance and ability to reliably indicate the } \\
\text { presence of pain and to reliably execute } \\
\text { instrumental tests }\end{array}$ & $\begin{array}{c}\text { Congenital craniofacial syndrome diagnosis } \\
\text { Presence of craniofacial bone fracture in the } \\
\text { medical history }\end{array}$ \\
& $\begin{array}{c}\text { Pr craniofacial surgery in the medical history } \\
\text { Presence of other systemic illness }\end{array}$ \\
\hline
\end{tabular}

The test group was compared with a control group composed of 32 healthy subjects without any signs or symptoms of JIA recruited from the Department of Orthodontics of the University of Chieti. These subjects did not need any orthodontic treatment until the end of the study and were matched for sex and age to the test group.

\subsection{Clinical Examination Methods}

All subjects underwent the first orthodontic and gnathologic visit by the same examiner. During the visit, for each patient, general medical history was extracted to assess other diseases, history of hospitalization and surgery, use of drugs, allergies and trauma. Dental medical history was also collected to assess oral health.

Head and neck muscle palpation was performed in all the subjects to take over the presence of muscular pain and trigger points. The anterior temporalis muscle (TA), masseter muscle (MM) and sternocleidomastoid muscle (SCM) on both sides were examined. Palpation was conducted by the same experienced examiner in a quiet room to obtain data without any emotional confounding factors. During muscle palpation, patients were asked to fill in a Visual Analogue Scale (VAS) $[28,29]$ indicating the pain intensity felt during the palpation of each muscle. Pain intensity was established by the patient with a value from 0 to 10 , where 0 means no pain and 10 means maximum pain. A value was reported for every muscle examined. The VAS score was filled in by each subject, without any influence from the operator.

\subsection{Recording Equipment}

The instrumental examination consisted of electromyography (sEMG) and kinesiography. Electromyographic activity was recorded using a wireless computerized instrument (Teethan BTS TMJOINT S.p.A. Garbagnate Milanese, MI, Italy), an innovative electromyograph technology using Dr. Ferrario's standardized protocols [30-33]. Six differential satellite surface electrodes with dimensions of $16 \times 12 \mathrm{~mm}$ and a mother electrode of $41.5 \times 24.8 \times 14 \mathrm{~mm}$ were used. The sEMG signals were amplified (gain 1000), with a bandwidth of $0-10 \mathrm{kHz}$ and peak-to-peak input range from 0 to $2000 \mu \mathrm{V}$, using a differential amplifier with a high common mode rejection ratio (CMMR $>110 \mathrm{~dB}$ in the range $50-60 \mathrm{~Hz}$, input impedance $100 \mathrm{MOhm}$ ), digitized (16b resolution; acquisition frequency up to $1 \mathrm{KHz}$ with $\pm 2 \%$ accuracy) and digitally filtered (high-pass filter set at $10 \mathrm{~Hz}$; low-pass filter set at $500 \mathrm{~Hz}$ ) [34]. The muscles investigated by sEMG were the right and left temporalis muscles (TA), the right and left masseter muscles (MM) and the right and left sternocleidomastoid muscles (SCM). Six adhesive electrodes were applied to the subjects' skin of the face and neck previously cleaned with alcohol to eliminate fat and pollution residues. The electrodes were placed on temporal, facial and neck areas where most of the recorded sEMG activity 
could be expected to come from the anterior temporalis, masseter and SCM muscles. In particular, on the anterior temporalis muscle area, the electrodes were positioned vertically, $3 \mathrm{~cm}$ from the zygomatic arch, just lateral to the eyebrow (lateral to the orbit of the eye); on the masseter muscle area, the electrodes were positioned between the cheek bone and the corner of the jaw [35]. On the sternocleidomastoid muscle area, one electrode was positioned in the middle of the sternal belly of the muscle. Data collection was conducted in a dental office by a single experienced examiner; the subject was seated on a comfortable chair with no headrest, with the feet resting on the floor and arms resting on the lap [35]. sEMG data were extracted with three tests lasting overall about $10 \mathrm{~min}$ :

Cotton test (COT6): The subject was asked to close their mouth and clench, biting two cotton rolls positioned on the occlusal surface of the posterior teeth behind the first premolar. This test allows assessing the maximum muscle activity in the absence of dental contact.

- Clenching test (CLE6): the subject was asked to close their mouth and clench in normal condition without cotton rolls (true occlusal test).

- $\quad$ Rotation test (ROT6): The subject was asked to rotate their head, first on one side, then on the other. The operator ensured that the patient did not tilt their head or move their shoulders away from the back of the chair.

For each subject, the maximum voluntary teeth clenching (MVC) value was extracted for MM and TA. The sEMG potentials recorded during the MVC tests were expressed as percent of the mean potential recorded during the MVC on the cotton rolls (unit IV/lV100). All following calculations were performed with the standardized potentials [36]. The ratio between MVC of masseter and temporal muscles (MM/TA) was used for evaluating each subject's muscle condition, even though the sEMG classical view does not provide for comparisons of the degree of force between different muscles [37]. According to Jankelson's studies [38], the MM/TA ratio under physiological conditions is greater than 1, while a value lower than 1 could point out pathological conditions in head and neck muscles. The pathological ratio is defined as an imbalance of temporalis and masseter muscles during their function.

Kinesiography was performed using the kinesiographer BioKeyNet (BioKet, S.Benedetto del Tronto, Italy) (Figure 1) [39,40], an electromedical instrument with a magnetic sensor detection system interfaced with a dedicated software able to detect, store and process all the movements of the jaw. The kinesiographic examination is non-invasive, short-lasting (15 min) and easy to carry out. It allows registering and showing in a graphic the jaw movements in the sagittal, frontal and transversal axes. The subject was seated, wearing a mask on their head calibrated on their anatomy using a ruler and a magnet placed on the lower frontal teeth. The following outcomes were registered: maximum mouth opening width and mandibular deviation at maximum mouth opening.
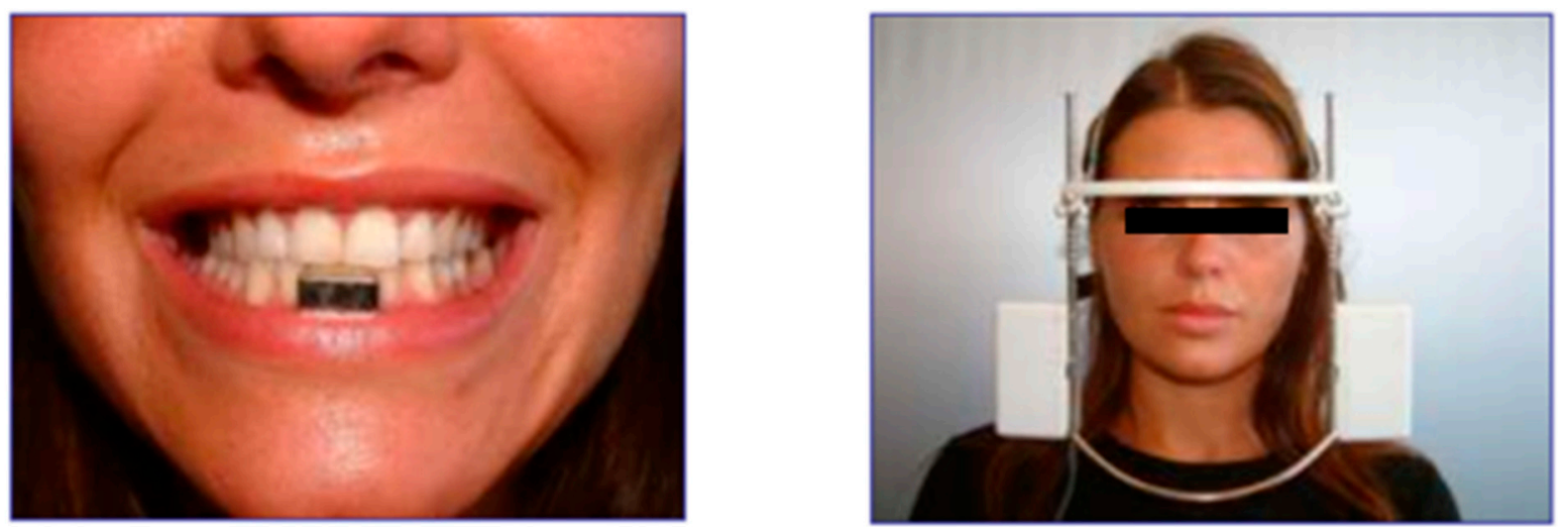

Figure 1. Kinesiography recording. A magnet was placed on the buccal surface of the lower incisors, and therefore a helmet with the sensors was positioned on the head of the subject with the help of a ruler. 


\subsection{Statistical Analysis}

Statistical analysis was performed using SPSS Statistics for Data Analysis v26. The Shapiro-Wilk normality test was applied to check whether data were normally distributed. Mean, standard deviation and median were calculated. Since data were not normally distributed, a non-parametric test, the Mann-Whitney U test, was used to compare the difference in muscle palpation using data extracted by VAS (Table 2) and kinesiography data between cases and the control group. Instead, the chi-squared test was used to verify any relation of the sEMG ratio (MM/TA) between the two groups. The differences were taken as significant when the $p$-value was less than 0.05 .

Table 2. Head and neck muscle palpation analysis (Mann-Whitney U test). Mean values of VAS (Visual Analogue Scale) resulting from palpation analysis on masseter, temporal and sternocleidomastoid muscles on both sides.

\begin{tabular}{cccc}
\hline Muscle & Group & Mean \pm SD & $p$-Value ${ }^{\text {a }}$ \\
\hline \multirow{2}{*}{ Right Sternocleidomastoid } & case & $3.44 \pm 3.02$ & \\
& control & $1.47 \pm 1.39$ & $0.009{ }^{*}$ \\
\hline \multirow{2}{*}{ Left Sternocleidomastoid } & case & $3.50 \pm 3.01$ & \\
& control & $1.47 \pm 1.39$ & $0.005^{*}$ \\
\hline Right Masseter & case & $1.03 \pm 1.15$ & \\
& control & $0.22 \pm 0.42$ & $0.002 *$ \\
\hline Left Masseter & & & \\
& case & $1.09 \pm 1.38$ & $0.049 *$ \\
\hline Right Temporal & control & $0.41 \pm 0.50$ & \\
& & & $0.018^{*}$ \\
\hline Left Temporal & case & $0.56 \pm 1.34$ & \\
& control & $0.06 \pm 0.25$ & 0.586 \\
\hline
\end{tabular}

Legend: SD: standard deviation; ${ }^{\text {a }}$ Mann-Whitney U test, ${ }^{*}$ level of significance was set at $p<0.05$.

\section{Results}

\subsection{Muscle Palpation Values}

Regarding MM and SCM muscles, higher pain was registered in the JIA group compared to the control group on both sides $(p<0.05)$. TA palpation values showed higher pain on the right side $(p<0.05)$, but not on the left side $(p>0.05)$, in the case group compared to controls (Table 2).

\section{2. sEMG Values}

The average MM/TA ratio was calculated in both study groups on both sides. It resulted that in the JIA group, the MM/TA ratio was lower than 1 on both sides ( 0.89 on right side and 0.90 on left side), thus indicating a pathological condition of the masticatory muscles according to Jankelson (pathological condition MM/TA < 1) [38]. On the contrary, the control group showed a physiological condition with an average ratio of 1.10 on the right side and 1.15 on the left side (physiological condition MM/TA > 1) (Table 3). 
Table 3. Head and neck electromyographic analysis (Mann-Whitney U test). Mean and standard deviation values of right and left masseter/temporal ratios (MM/TA) in case and control groups.

\begin{tabular}{cccc}
\hline Muscle & Group & Mean \pm SD & $p$-Value ${ }^{\text {a }}$ \\
\hline Right MM/TA & case & $0.89 \pm 0.37$ & \\
& control & $1.10 \pm 0.60$ & 0.170 \\
\hline \multirow{2}{*}{ Left Sternocleidomastoid } & case & $0.41 \pm 0.84$ & \\
& control & $0.69 \pm 0.97$ & 0.122 \\
\hline
\end{tabular}

Legend: SD: standard deviation; MM/TA = masseter/temporal ratio. ${ }^{\text {a }}$ Mann-Whitney U test, level of significance was set at $p<0.05$.

The test group showed a higher frequency of the pathological ratio $(65.2 \%)$ compared with the control group (53\%). The chi-squared test was used to verify if the frequency of pathological ratio of $\mathrm{MM}$ and TA $(\mathrm{MM} / \mathrm{TA}<1)$ was significantly higher in the JIA group than in the control group. The test showed a positive correlation between the pathological ratio $(\mathrm{MM} / \mathrm{TA}<1)$ and JIA, with an $\chi^{2}$ value of 2.006 , but no statistically significant difference was found $(p=0.157)$ (Table 4$)$.

Table 4. Electromyographic analysis (chi-squared test). Frequencies of pathological and physiological ratios in case and control groups.

\begin{tabular}{cccccc}
\hline Group & Frequency & $\begin{array}{c}\text { Percentage of } \\
\text { Frequency }\end{array}$ & $\chi^{2}$ Value & $p$-Value ${ }^{\text {a }}$ \\
\hline \multirow{2}{*}{ case } & $\begin{array}{c}\text { Pathological ratios } \\
\text { Physiological } \\
\text { ratios }\end{array}$ & 43 & $65.2 \%$ & & \\
& 23 & $34.8 \%$ & & \\
\hline \multirow{2}{*}{ control } & $\begin{array}{c}\text { Pathological ratios } \\
\text { Physiological } \\
\text { ratios }\end{array}$ & 35 & $53.0 \%$ & & \\
& & 31 & $47.0 \%$ & 2.006 & 0.157 \\
\hline
\end{tabular}

\subsection{Kinesiographic Values}

The Mann-Whitney $U$ test was used to compare the maximum mouth opening width and jaw deviation at maximum mouth opening. A statistically significant result $(p<0.05)$ was found comparing the average maximum mouth opening in the JIA group $(35.88 \pm 6.92 \mathrm{~mm})$ and the control group $(40.12 \pm 1.58 \mathrm{~mm})$. Regarding jaw deviation at mouth opening, the average in the JIA group was $5.69 \pm 3.06 \mathrm{~mm}$, while in the control group, it was $4.18 \pm 1.89 \mathrm{~mm}$. A statistically significant difference was found $(p<0.05)$ between the two groups. A total of 29 out of 32 JIA subjects showed jaw deviation towards the right side (Table 5).

Table 5. Kinesiographic analysis (Mann-Whitney U test). Mean and standard deviation values of mouth opening and deviation on mouth opening calculated in millimeters $(\mathrm{mm})$ in case and control groups.

\begin{tabular}{cccc}
\hline & Group & Mean \pm SD & $p$-Value ${ }^{\text {a }}$ \\
\hline \multirow{2}{*}{ Mouth opening $(\mathrm{mm})$} & case & $35.89 \pm 6.93$ & \\
& control & $40.13 \pm 1.58$ & $0.001^{*}$ \\
\hline \multirow{2}{*}{ Mouth opening deviation $(\mathrm{mm})$} & case & $5.70 \pm 3.06$ & \\
& control & $4.20 \pm 1.90$ & $0.024^{*}$ \\
\hline
\end{tabular}

Legend: SD: standard deviation; ${ }^{\text {a }}$ Mann-Whitney U test, ${ }^{*}$ level of significance was set at $p<0.05$. 


\section{Discussion}

The present study investigated parameters assessed by clinical head and neck muscle palpation and instrumental examinations by sEMG and kinesiography in the evaluation of TMJ involvement in patients with JIA. These tools do not replace the gold standard of diagnosis, which remains nuclear magnetic resonance imaging (MRI) [41], but may help clinicians to identify patients at higher risk of TMJ involvement and those who are more likely to benefit from screening or early therapeutic intervention [42-44]. Muscle palpation, sEMG and kinesiography can therefore be considered functional indicators of muscle activity and jaw movements since they show the tendency to support experimental hypotheses. The presence, however, of statistically significant parameters confirms the possibility of demonstrating the validity of these diagnostic aids.

Muscle palpation analysis (Table 2) showed a statistically significant difference between the JIA group and the control group in the MM and SCM evaluation on both sides $(p<0.05)$ and in the TA only on the right side $(p<0.05)$. Pain on palpation of the orofacial regions is one of the clinical signs detected by a task force investigating clinical orofacial examination in JIA [24], in agreement with the results of our study. These results indicate that there is higher pain in the masticatory and neck muscles in the JIA group compared to healthy controls $[45,46]$. Due to condylar erosion, masticatory muscles force the mandibular bone to rotate backwards with respect to the anterior skull base, thus generating dentoalveolar deformities. This might cause an overload of the masticatory muscles (which become painful on palpation) and of the joint structures, leading to muscle imbalances and possible intracapsular disorders. On the left TA, higher pain was recorded in the JIA group compared to the control group; statistical significance, however, was lacking, probably due to the low sample size. Another explanation of these results might be found in the prevalence of jaw deviation on the right side observed in the JIA group, where, indeed, 29 out of 32 JIA subjects showed jaw deviation towards the right side. Finally, the significant results found in SCM highlight the link existing between chewing muscles and neck muscles and therefore the need to take into consideration the involvement of neck muscles in addition to chewing muscles in patients with JIA [47].

Statistical analysis of sEMG data showed a positive correlation between the pathological ratio (MM/TA < 1) and JIA, with an $\chi^{2}$ value of 2.006 (Table 4). However, since this correlation was present, but it was not statistically significant $(p=0.157)$, frequencies in terms of percentage were calculated. A higher frequency of the pathological ratio $(\mathrm{MM} \backslash \mathrm{TA}<1)$ in the test group $(65.2 \%)$ compared with the control group (53\%) was observed. The prevalence of the pathological ratio in the group of patients indicates that JIA subjects showed an incorrect function of the masticatory muscle system. The masticatory muscle alteration observed in sEMG data confirmed the results extracted from palpation, where greater pain was observed in the JIA group. The progressive erosion suffered by TMJ structures in JIA patients involves masticatory muscles which must readapt the masticatory pattern to ensure the function of the stomatognathic system. This adaptation leads to muscle dysfunction and pain, as shown by sEMG and muscle palpation data. It is therefore reasonable to assume that if no intervention on the stomatognathic system is performed, muscle alteration and pain could progressively become worse as the erosion on TMJ structures continues [48-50].

Kinesiographic statistical analysis (Table 5) showed a statistically significant difference both in the maximum mouth opening width and jaw deviation at maximum mouth opening between cases and controls.

Regarding mouth opening, the results of the present work disagree with a previous study by Koos et al. [51] which investigated the reliability of clinical examinations such as mouth opening and deflection during mouth opening to detect TMJ arthritis. The study concluded that a reduced mouth opening capacity might not always be a very reliable criterion for diagnosing TMJ arthritis. However, the authors stated further studies are needed to evaluate if this parameter could be considered as a reliable criterion for monitoring the pathology evolution in the same subject [51]. 
For the maximum opening deviation parameter, statistical significance was found, in accordance with what was stated in the literature by Koos et al. [51]. Mouth opening limitations and deflections may be related to dysfunctions of the TMJ. In addition, another study by Rongo et al. [52] confirmed that chin deviation, TMJ crepitus and reduction in mouth opening are associated with a high risk of TMJ involvement and are more frequent when the damage of the TMJ is more severe. The authors suggested combining pain-related variables with function-related variables to achieve a higher diagnostic performance in diagnosing a TMJ involvement in JIA patients [52,53].

Since a statistically significant difference between the kinesiographic parameters of the two groups under investigation was found, they might help clinicians to find small and early joint abnormalities due to their sensibility which greatly surpasses that of clinical methods $[54,55]$. For instance, the prevalence of jaw deviation towards the right side observed in the JIA group might explain why the left temporal muscle is not statistically significant in muscle palpation analysis. Jaw deviation is directed to the side who showed more condylar erosion; thus, it can be supposed that TMJ erosions were worse on the right side than the left side: condylar erosion on the right side forces muscles of the right side to work harder to bring the head of the condyle closer to the mandibular fossa. On the other hand, muscles on the left side have less workload and this could explain why they did not show statistically significant results on muscle palpation analysis.

sEMG, kinesiography and muscle palpation data showed a greater alteration of the stomatognathic system. This could be explained considering that sEMG and muscle palpation are muscular pain indicators while kinesiography evaluates joint structure alterations on jaw movements. As stated by Okeson [47], muscular suffering is an earlier predictive sign of TMJ disorders while joint damage occurs later. In addition, kinesiography analysis might reveal signs of more advanced involvement of the TMJ in JIA, while sEMG could detect TMJ damage in an earlier stage. This suggests the importance of muscle evaluation in growing patients to detect an early involvement of the joint structures. Therefore, it is possible to emphasize the relevance to recognize clinical symptoms indicating TMJ involvement in an early stage to avoid JIA progressive lesions. The association of clinical muscle palpation, instrumental evaluation by kinesiography and radiological abnormalities may lead to early detection of imminent condylar erosion.

\section{Limitation}

This study evaluated patients attending a single center and analyzed at a single point in time. Further longitudinal investigations are needed to scrutinize the relative ability of the various clinical and instrumental items to assess an involvement of the TMJ.

A wider test group should be considered in order to make the collected data fully reliable and overcome the difficulties related to carrying out tests in poorly compliant young subjects.

\section{Conclusions}

This experimental study showed that JIA affects the TMJ, causing myalgia in the head and neck muscles, a reduction in mouth opening width and an increase in jaw deviation during mouth opening. Further studies involving MRI imaging are needed to confirm TMJ arthritic involvement in patients showing JIA in other body districts.

Author Contributions: Conceptualization, M.D., B.D.C. and L.B.; methodology, M.D., B.D.C., F.C. (Francesco Caroccia) and F.M.; software, B.D.C.; validation, M.D., F.C. (Francesco Caroccia), F.M. and L.B.; formal analysis, F.C. (Francesco Caroccia) and F.M.; investigation, M.D., B.D.C. and D.M.d.; resources, M.D. and L.B.; data curation, B.D.C.; writing-original draft preparation, B.D.C.; writingreview and editing, F.C. (Francesco Caroccia), F.M. and D.M.d.; supervision, F.C. (Francesco Chiarelli) and F.F. All authors have read and agreed to the published version of the manuscript.

Funding: This research received no external funding. 
Institutional Review Board Statement: This study was conducted according to the guidelines of the Declaration of Helsinki and approved by the Institutional Review Board (or Ethics Committee) of the University of Chieti (protocol code MGB_AIG).

Informed Consent Statement: Informed consent was obtained from all subjects involved in the study.

Data Availability Statement: The data presented in this study are available on request from the corresponding author.

Conflicts of Interest: The authors declare no conflict of interest.

\section{References}

1. Crayne, C.B.; Beukelman, T. Juvenile Idiopathic Arthritis: Oligoarthritis and Polyarthritis. Pediatr. Clin. N. Am. 2018, 65, 657-674. [CrossRef] [PubMed]

2. Cimaz, R. Systemic-onset juvenile idiopathic arthritis. Autoimmun. Rev. 2016, 15, 931-934. [CrossRef] [PubMed]

3. Niibo, P.; Pruunsild, C.; Voog-Oras, Ü.; Nikopensius, T.; Jagomägi, T.; Saag, M. Contemporary management of TMJ involvement in JIA patients and its orofacial consequences. EPMA J. 2016, 7, 12. [CrossRef]

4. Thatayatikom, A.; De Leucio, A. Juvenile Idiopathic Arthritis (JIA); StatPearls Publishing: Treasure Island, FL, USA, 2020.

5. Horton, D.B.; Shenoi, S. Review of environmental factors and juvenile idiopathic arthritis. Open Access Rheumatol. 2019, 11, 253-267. [CrossRef] [PubMed]

6. Giancane, G.; Alongi, A.; Ravelli, A. Update on the pathogenesis and treatment of juvenile idiopathic arthritis. Curr. Opin. Rheumatol. 2017, 29, 523-529. [CrossRef]

7. Mahmud, S.A.; Binstadt, B.A. Autoantibodies in the Pathogenesis, Diagnosis, and Prognosis of Juvenile Idiopathic Arthritis. Front. Immunol. 2019, 9, 3168. [CrossRef] [PubMed]

8. Barut, K.; Adrovic, A.; Şahin, S.; Kasapçopur, Ö. Juvenile Idiopathic Arthritis. Balkan Med. J. 2017, 34, 90-101. [CrossRef] [PubMed]

9. Petty, R.E.; Southwood, T.R.; Baum, J.; Bhettay, E.; Glass, D.N.; Manners, P.; Maldonado-Cocco, J.; Suarez-Almazor, M.; Orozco-Alcala, J.; Prieur, A.M. Revision of the proposed classification criteria for juvenile idiopathic arthritis: Durban, 1997. J. Rheumatol. 1998, 25, 1991-1994.

10. Petty, R.E.; Southwood, T.R.; Manners, P.; Baum, J.; Glass, D.N.; Goldenberg, J.; He, X.; Maldonado-Cocco, J.; Orozco-Alcala, J.; Prieur, A.-M.; et al. International League of Associations for Rheumatology classification of juvenile idiopathic arthritis: Second revision, Edmonton, 2001. J. Rheumatol. 2004, 31, 390-392.

11. Martini, A.; Ravelli, A.; Avcin, T.; Beresford, M.W.; Burgos-Vargas, R.; Cuttica, R.; Ilowite, N.T.; Khubchandani, R.; Laxer, R.M.; Lovell, D.J.; et al. Toward New Classification Criteria for Juvenile Idiopathic Arthritis: First Steps, Pediatric Rheumatology International Trials Organization International Consensus. J. Rheumatol. 2019, 46, 190-197. [CrossRef]

12. Malattia, C.; Rinaldi, M.; Martini, A. The role of imaging in juvenile idiopathic arthritis. Expert Rev. Clin. Immunol. 2018, 14, 681-694. [CrossRef] [PubMed]

13. Merle, C.L.; Hoffmann, R.; Schmickler, J.; Rühlmann, M.; Challakh, N.; Haak, R.; Schmalz, G.; Ziebolz, D. Comprehensive Assessment of Orofacial Health and Disease Related Parameters in Adolescents with Juvenile Idiopathic Arthritis-A CrossSectional Study. J. Clin. Med. 2020, 9, 513. [CrossRef]

14. Billiau, A.D.; Hu, Y.; Verdonck, A.; Carels, C.; Wouters, C. Temporomandibular joint arthritis in juvenile idiopathic arthritis: Prevalence, clinical and radiological signs, and relation to dentofacial morphology. J. Rheumatol. 2007, 34, 1925-1933. [PubMed]

15. Steenks, M.H.; Giancane, G.; de Leeuw, R.R.; Bronkhorst, E.M.; van Es, R.J.J.; Koole, R.; van Bruggen, H.W.; Wulffraat, N.M. Temporomandibular joint involvement in Juvenile Idiopathic Arthritis: Reliability and validity of a screening protocol for the rheumatologist. Pediatr. Rheumatol. 2015, 13, 15. [CrossRef] [PubMed]

16. Kjellberg, H. Juvenile chronic arthritis. Dentofacial morphology, growth, mandibular function and orthodontic treatment. Swed Dent. J. Suppl. 1995, 109, 1-56. [PubMed]

17. Cedströmer, A.-L.; Andlin-Sobocki, A.; Abbu, N.; Hedenberg-Magnusson, B.; Dahlström, L.; Berntson, L. Condylar alterations and facial growth in children with juvenile idiopathic arthritis. J. Orofac. Orthop. 2020, 81, 163-171. [CrossRef] [PubMed]

18. Svensson, B.; Adell, R.; Kopp, S. Temporomandibular disorders in juvenile chronic arthritis patients. A clinical study. Swed Dent. J. 2000, 24, 83-92.

19. Bernini, J.M.; Kellenberger, C.J.; Eichenberger, M.; Eliades, T.; Papageorgiou, S.N.; Patcas, R. Quantitative analysis of facial asymmetry based on three-dimensional photography: A valuable indicator for asymmetrical temporomandibular joint affection in juvenile idiopathic arthritis patients? Pediatr. Rheumatol. Online J. 2020, 18, 10. [CrossRef]

20. Zwir, L.F.; Terreri, M.T.; Castro, A.D.A.; Rodrigues, W.D.R.; Fernandes, A.R.C. Is power Doppler ultrasound useful to evaluate temporomandibular joint inflammatory activity in juvenile idiopathic arthritis? Clin. Rheumatol. 2020, 39, 1237-1240. [CrossRef]

21. Hsieh, Y.-J.; Darvann, T.A.; Hermann, N.V.; Larsen, P.; Liao, Y.-F.; Kreiborg, S. Three-dimensional assessment of facial morphology in children and adolescents with juvenile idiopathic arthritis and moderate to severe TMJ involvement using 3D surface scans. Clin. Oral Investig. 2020, 24, 799-807. [CrossRef]

22. Urtane, I.; Jankovska, I.; Al-Shwaikh, H.; Krisjane, Z. Correlation of temporomandibular joint clinical signs with cone beam computed tomography radiologic features in juvenile idiopathic arthritis patients. Stomatologija 2018, 20, 82-89. 
23. Klenke, D.; Quast, A.; Prelog, M.; Holl-Wieden, A.; Riekert, M.; Stellzig-Eisenhauer, A.; Meyer-Marcotty, P. TMJ pathomorphology in patients with JIA-radiographic parameters for early diagnosis. Head Face Med. 2018, 14, 15. [CrossRef]

24. Stoustrup, P.; Twilt, M.; Spiegel, L.; Kristensen, K.D.; Koos, B.; Pedersen, T.K.; Küseler, A.; Cron, R.Q.; Abramowicz, S.; Verna, C.; et al. Clinical Orofacial Examination in Juvenile Idiopathic Arthritis: International Consensus-based Recommendations for Monitoring Patients in Clinical Practice and Research Studies. J. Rheumatol. 2017, 44, 326-333. [CrossRef]

25. Stoll, M.L.; Kau, C.H.; Waite, P.D.; Cron, R.Q. Temporomandibular joint arthritis in juvenile idiopathic arthritis, now what? Pediatr. Rheumatol. Online J. 2018, 16, 32. [CrossRef] [PubMed]

26. Kristensen, K.D.; Stoustrup, P.; Küseler, A.; Pedersen, T.K.; Twilt, M.; Herlin, T. Clinical predictors of temporomandibular joint arthritis in juvenile idiopathic arthritis: A systematic literature review. Semin. Arthritis Rheum. 2016, 45, 717-732. [CrossRef] [PubMed]

27. Von Elm, E.; Altman, D.G.; Egger, M.; Pocock, S.J.; Gøtzsche, P.C.; Vandenbroucke, J.P. STROBE Initiative. The Strengthening the Reporting of Observational Studies in Epidemiology (STROBE) statement: Guidelines for reporting observational studies. J. Clin. Epidemiol. 2008, 61, 344-349. [CrossRef] [PubMed]

28. Sung, Y.-T.; Wu, J.-S. The Visual Analogue Scale for Rating, Ranking and Paired-Comparison (VAS-RRP): A new technique for psychological measurement. Behav. Res. Methods 2018, 50, 1694-1715. [CrossRef] [PubMed]

29. Goddard, G.; Karibe, H.; McNeill, C. Reproducibility of visual analog scale (VAS) pain scores to mechanical pressure. Cranio 2004, 22, 250-256. [CrossRef]

30. Ferrario, V.F.; Tartaglia, G.M.; Luraghi, F.E.; Sforza, C. The use of surface electromyography as a tool in differentiating temporomandibular disorders from neck disorders. Man Ther. 2007, 12, 372-379. [CrossRef] [PubMed]

31. Ferrario, V.F.; Sforza, C.; Serrao, G. The influence of crossbite on the coordinated electromyographic activity of human masticatory muscles during mastication. J. Oral Rehabil. 1999, 26, 575-581. [CrossRef]

32. Ferrario, V.F.; Tartaglia, G.M.; Galletta, A.; Grassi, G.P.; Sforza, C. The influence of occlusion on jaw and neck muscle activity: A surface EMG study in healthy young adults. J. Oral Rehabil. 2006, 33, 341-348. [CrossRef]

33. De Felìcio, C.M.; Sidequersky, F.V.; Tartaglia, G.M.; Sforza, C. Electromyographic standardized indices in healthy Brazilian young adults and data reproducibility. J. Oral Rehabil. 2009, 36, 577-583. [CrossRef]

34. Teethan User Manual. Document Number: ERTHN-01376-00 Published: February 2016 Copyright (C 2014-2015-2016 Teethan S.p.A. All Rights Reserved. Available online: https://teethan.com/immagini/TEETHAN_User_Manual_02-07-2019_LQ_ENG.pdf (accessed on 10 February 2021).

35. Berni, K.C.; Dibai-Filho, A.V.; Pires, P.F.; Rodrigues-Bigaton, D. Accuracy of the surface electromyography RMS processing for the diagnosis of myogenous temporomandibular disorder. J. Electromyogr. Kinesiol. 2015, 25, 596-602. [CrossRef]

36. Augusti, D.; Augusti, G.; Re, D.; Dellavia, C.; Giannì, A.B. Effect of different dental articulating papers on SEMG activity during maximum clenching. J. Electromyogr. Kinesiol. 2015, 25, 612-618. [CrossRef] [PubMed]

37. Basmajian John, V.; De Luca, C.J. Muscles Alive. Their Functions Relieved by Electromyography, 5th ed.; William/Wilkin: Baltimore, MD, USA, 1985.

38. Jankelson, B. Neuromuscular aspects of occlusion. Effects of occlusal position on the physiology and dysfunction of the mandibular musculature. Dent. Clin. N. Am. 1979, 23, 157-168.

39. Cesaretti, G.; Gobbi, G. Kinesiografia e sEMG su Pazienti Affetti da Bruxismo; Bioket: San Benedetto del Tronto, Italy, 2013.

40. Papini, A.; Cesaretti, G.; Defabianis, P. Kinesiographic analysis of lateral excursive movement on the horizontal plane: The retrusive component. J. Oral Sci. Rehabil. 2017, 3, 60-67.

41. Müller, L.; Kellenberger, C.J.; Cannizzaro, E.; Ettlin, D.; Schraner, T.; Bolt, I.B.; Peltomäki, T.; Saurenmann, R.K. Early diagnosis of temporomandibular joint involvement in juvenile idiopathic arthritis: A pilot study comparing clinical examination and ultrasound to magnetic resonance imaging. Rheumatology 2009, 48, 680-685. [CrossRef]

42. Pedersen, T.K.; Küseler, A.; Gelineck, J.; Herlin, T. A prospective study of magnetic resonance and radiographic imaging in relation to symptoms and clinical findings of the temporomandibular joint in children with juvenile idiopathic arthritis. J. Rheumatol. 2008, 35, 1668-1675.

43. Lucchese, A.; Carinci, F.; Brunelli, G.; Monguzzi, R. Everstick ${ }^{\circledR}$ and Ribbond ${ }^{\circledR}$ fiber reinforced composites: Scanning Electron Microscope (SEM) comparative analysis. Eur. J. Inflamm. 2011, 3, 73-79.

44. Roncati, M.; Polizzi, E.; Cingano, L.; Gherlone, E.F.; Lucchese, A. An oral health aid for disabled patients. Dent. Cadmos 2013, 81, 447-452. [CrossRef]

45. Stoustrup, P.; Kristensen, K.D.; Verna, C.; Küseler, A.; Herlin, T.; Pedersen, T.K. Orofacial symptoms related to temporomandibular joint arthritis in juvenile idiopathic arthritis: Smallest detectable difference in self-reported pain intensity. J. Rheumatol. 2012, 39, 2352-2358. [CrossRef] [PubMed]

46. Woźniak, K.; Lipski, M.; Lichota, D.; Szyszka-Sommerfeld, L. Muscle Fatigue in the Temporal and Masseter Muscles in Patients with Temporomandibular Dysfunction. BioMed Res. Int. 2015, 2015, e269734. [CrossRef] [PubMed]

47. Jeffrey, O. Management of Temporomandibular Disorders and Occlusion, 7th ed.; Mosby: St. Louis, MO, USA, 2013.

48. Bakke, M.; Zak, M.; Jensen, B.L.; Pedersen, F.K.; Kreiborg, S. Orofacial pain, jaw function, and temporomandibular disorders in women with a history of juvenile chronic arthritis or persistent juvenile chronic arthritis. Oral Surg. Oral Med. Oral Pathol. Oral Radiol. Endod. 2001, 92, 406-414. [CrossRef] [PubMed] 
49. Baena, R.R.; Pastorino, R.; Gherlone, E.F.; Perillo, L.; Lupi, S.M.; Lucchese, A. Histomorphometric Evaluation of two Different Bone Substitutes In Sinus Augumentation Procedures: A Randomized Controlled Trial in Humans. Int. J. Oral Maxillofac. Implants. 2017, 32, 188-194. [CrossRef]

50. Matarese, G.; Isola, G.; Ramaglia, L.; Dalessandri, D.; Lucchese, A.; Alibrandi, A.; Fabiano, F.; Cordasco, G. Periodontal biotype: Characteristic, prevalence and dimensions related to dental malocclusion. Minerva Stomatol. 2016, 65, 231-238.

51. Koos, B.; Twilt, M.; Kyank, U.; Fischer-Brandies, H.; Gassling, V.; Tzaribachev, N. Reliability of Clinical Symptoms in Diagnosing Temporomandibular Joint Arthritis in Juvenile Idiopathic Arthritis. J. Rheumatol. 2014, 41, 1871-1877. [CrossRef] [PubMed]

52. Rongo, R.; Alstergren, P.; Ammendola, L.; Bucci, R.; Alessio, M.; D'Antò, V.; Michelotti, A. Temporomandibular joint damage in juvenile idiopathic arthritis: Diagnostic validity of diagnostic criteria for temporomandibular disorders. J. Oral Rehabil. 2019, 46, 450-459. [CrossRef]

53. Manuelli, M. A peaceful man. Prog. Orthod. 2012, 13, 1. [CrossRef]

54. Mohl, N.D. Reliability and validity of diagnostic modalities for temporomandibular disorders. Adv. Dent. Res. 1993, 7, 113-119. [CrossRef]

55. Bertossi, D.; Giampaoli, G.; Lucchese, A.; Manuelli, M.; Albanese, M.; Nocini, R.; Nocini, P.F. The skin rejuvenation associated tratment-Fraxel laser, Microbotox, and low G prime hyaluronic acid: Preliminary results. Laser Med. Sci. 2019, 34, 1449-1455. [CrossRef] 